\title{
Alteraciones en la tasa de respiración de Daphnia magna bajo concentraciones subletales de anatoxina-a.
}

\author{
Rodrigues da Silva, S.L1,3, D. Oliveira Rodrigues ${ }^{1,4}$, P. Sánchez Castillo ${ }^{2}$, \\ J.M. Conde-Porcuna ${ }^{1}$ y L. Cruz-Pizarro ${ }^{1}$. \\ ${ }^{1}$ Instituto del Agua, Universidad de Granada. C/ Ramón y Cajal, 04, 18071, Granada. España. \\ ${ }^{2}$ Departamento de Biología Vegetal, Facultad de Ciencias, Universidad de Granada, C/ Severo Ochoa, s/n, \\ 18071, Granada. España. \\ ${ }^{3}$ Departamento de Ecotoxicologia, Universidade Santa Cecília. Rua Oswaldo Cruz, 266, 11045-907, \\ Santos. Brasil. \\ ${ }^{4} \mathrm{CNPq}$, Brasil.
}

\section{RESUMEN}

Anatoxina-a es una potente neurotoxina producida por cianobacterias dulceacuícolas que puede representar un riesgo, por sus efectos agudos, para diferentes formas de vida, incluyendo los seres humanos. Aunque se conocen los efectos agudos y crónicos de esta toxina, pocos estudios aportan datos de medidas de efectos subletales sobre la respiración del zooplancton. En este trabajo se cuantifican cambios en la respiración de Daphnia magna bajo una concentración subletal de anatoxina-a, en condiciones experimentales, por medio de un sistema automatizado de medida en continuo de consumo de Oxígeno. Los resultados indican que la presencia de anatoxina-a en dosis subletales reduce significativamente la actividad respiratoria en esta especie, con efectos, dependientes de la edad del organismo.

Palabras clave: Cianobacterias, anatoxina-a, Daphnia, respiración.

\begin{abstract}
Anatoxin-a is a potent neurotoxin produced by some freshwater cyanobacteria which, because of its acute toxic effects, may represent a hazard to aquatic organisms, and even to human beings. Both the acute and chronic effects of this toxin are rather well known, but few studies provided information about its sublethal effect on the zooplankton respiration. In this paper we have quantified respiration changes in Daphnia magna under sublethal anatoxin-a concentrations in experimental conditions, using an automatic open-flow system of continuous measurement of oxygen consumption. Our results show that the presence of anatoxin-a at sublethal doses reduces significantly the respiratory activity of the animals, being the effects greatly dependent on the individual age.
\end{abstract}

Keywords: Cyanobacteria, anatoxin-a, Daphnia, respiration.

\section{INTRODUCCIÓN}

Muchos ecosistemas eutróficos o sometidos a procesos de eutrofización se caracterizan por un desarrollo estacional masivo (blooms) de cianobacterias, en un marco de características físico-químicas, hidrodinámicas y meteorológicas definidas (p.ej. Sivonen, 1998; Barros et al., 2001; Cruz-Pizarro et al., 2002), que en el caso de sistemas destinados al abastecimiento, suelen crear severos problemas, bien por la modificación del medio, bien por la producción de sustancias malolientes y/o tóxicas. Se ha podido evidenciar que la morfología, composición química y toxicidad de las cianobacterias interfieren negativamente con la alimentación, nutrición, supervivencia, reproducción y crecimiento del zooplancton, especialmente de los grandes cladóceros (Fulton \& Paerl, 1987; Davidowicz, et al., 1988; Gliwicz \& 
Lampert, 1990; Barros, et al., 2001) y, de hecho, algunas de estas características se han considerado como defensas frente al consumo herbívoro desarrolladas por estos organismos (Lampert, 1987).

Dentro de las especies de cianobacterias, destacan aquellas productoras de toxinas, especialmente los géneros Anabaena, Microcystis, Aphanizomenon, Oscillatoria, Nodularia y Cylindrospermopsis (Sivonen, 1998; Vasconcelos, 2001).

Las toxinas de cianobacterias, se suelen clasificar en tres grupos, en función de sus efectos sobre los mamíferos: irritantes, neurotoxinas y hepatotoxinas (Vasconcelos, 2001). La primera no suele representar un elevado riesgo para la salud. Sin embargo las hepatotoxinas causan graves alteraciones en los hepatocitos pudiendo ocasionar la letalidad en algunas horas o insuficiencia hepática seguida de muerte al final de algunos días (Carmichael, 1994; Barros, 1994). Las neurotoxinas bloquean los neurotransmisores y causan muerte rápida por parálisis del sistema respiratorio (Sivonen, 1998).

Una primera revisión bibliográfica no ha permitido encontrar información sobre alteraciones de la tasa de respiración en el zooplancton en presencia de cianotoxinas, posiblemente debido a la dificultad en medir esta variable bajo condiciones de control experimental. Asimismo, no se dispone de estudios previos que traten de analizar diferentes respuestas toxicológicas de juveniles y adultos de Daphnia en presencia de cianotoxinas.

En este estudio, se ha analizado el efecto de una potente neurotoxina, la anatoxina-a, sobre la tasa de respiración de individuos de diferentes edades del género Daphnia. Dicha toxina se utilizó en concentraciones muy bajas, previamente establecidas de acuerdo con un análisis experimental preliminar y por estudios anteriores (p.ej. Claska \& Gilbert, 1998). Además, el hecho de que el género Daphnia sea ampliamente utilizado en tests de toxicidad y de interacción con cianobacterias, permitirá la posibilidad de comparar los resultados obtenidos en este estudio con aspectos fisiológicos, previamente descritos en otros estudios.

\section{MATERIAL Y MÉTODOS}

\section{Organismo-test}

Como organismo-test hemos utilizado individuos de Daphnia magna Straus, 1820 (Cladocera, Crustacea), identificados y aislados de muestras de zooplancton de la laguna Honda (Albufera de Adra, Almería, España). Se adoptó un procedimiento de cultivo basado en normas españolas e internacionales (AENOR, 1996; ISO 6341, 1982), utilizando como fuente de alimento células de Chlorella vulgaris, cultivadas según se describe en las normas de la ABNT ("Associação Brasileira de Normas Técnicas") - NBR 12713 (1993) y ABNT - NBR 12648 (1992). Los cultivos (80 100 individuos), mantenidos en medio M4 (Elendt \& Bias, 1990), bajo condiciones controladas de temperatura $\left(20{ }^{\circ} \mathrm{C}\right)$, luz (fluorescente con densidad de flujo de $48.1 \mu \mathrm{mol} \mathrm{s} \mathrm{s}^{-1} \mathrm{~m}^{-2}$ ) y fotoperiodo (16/8 h luz/oscuridad)), fueron alimentados diariamente con aproximadamente $3 \mathrm{ml}$ de solución de Chlorella vulgaris con una concentración celular de aproximadamente $2 \cdot 10^{7}$ cells $/ \mathrm{ml}$.

\section{Anatoxina-a}

Suministrada en estado puro por "Sigma-Aldrich Química, S.A." Presenta la fórmula molecular $\mathrm{C}_{10} \mathrm{H}_{15} \mathrm{NO}$ y recibe las siguientes denominaciones químicas: (1R)-1-(9-Azabicyclo[4.2.1.]non-2-en-2yl)-ethanone; (+)-2-Acetyl-9-azabicyclo(4.2.1)non2-ene o $(+)$-AnTx-a. En la figura 1, se presenta la estructura química, tal y como se describe por Research Biochemicals International $\left(\mathrm{RBI}^{\circledR}\right)$.

\section{Diseño experimental}

Se realizó un test preliminar de toxicidad (AENOR, 1996; ISO 6341, 1982), que se utilizó como referencia para reconocimiento de los niveles de toxicidad aguda de la cianotoxina con $D$. magna. Para la realización de dicho test, se prepararon 7 concentraciones en el rango de 1 a $2000 \mu \mathrm{g} / \mathrm{l}$ de anatoxina-a, utilizando como agua de dilución el medio M4. Para cada concentración, además de un control (solamente con medio 


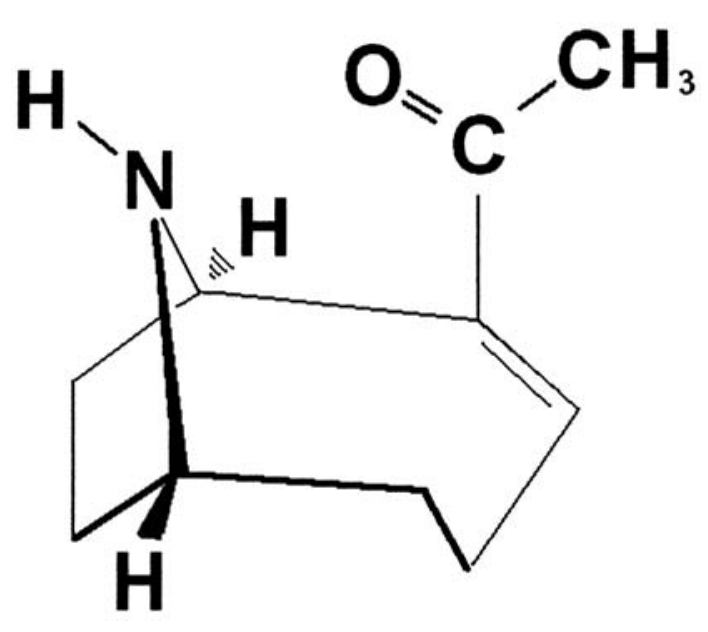

Figura 1. Estructura química de la anatoxina-a (según Research Biochemicals International - $\left.\mathrm{RBI}^{\circledR}\right)$. The chemical structure of Anatoxin-a (according to the Research Biochemicals International - $\left.R B I^{\circledR}\right)$.

M4), fueron preparadas 4 réplicas con volumen de $10 \mathrm{ml}$, y en cada una fueron puestos 5 neonatos de D. magna (20 organismos por concentración). El ensayo tuvo la duración de 24 horas y se mantuvo en cámara de germinación con temperatura de $20^{\circ} \mathrm{C}+/-1^{\circ} \mathrm{C}$, en estado de ayuno y en oscuridad. Al final del período del ensayo, se realizaron los recuentos de organismos móviles e inmóviles (son considerados inmóviles aquellos incapaces de desplazarse en los 15 segundos siguientes a una ligera agitación del recipiente, aunque puedan mover sus antenas).

Se adoptaron los siguientes tratamientos de medidas de consumo de oxígeno: un tratamiento de control, en el que se utilizó solamente el medio M4 y un tratamiento con concentración subletal (establecida con base en el test preliminar de toxicidad) de anatoxina-a. Estos dos tratamientos, a su vez, se repitieron para dos edades: juveniles (con edad inferior a 48 horas) y organismos adultos con edad superior a 21 días. Cada tratamiento se replicó 4 veces.

En la medida del consumo de oxígeno se utilizó un respirómetro de flujo continuo y sistema abierto (Cyclobios - Twin-Flow Respirometer) con dos sensores polarográficos (POS). El sistema consiste de 6 módulos: una unidad electróni-

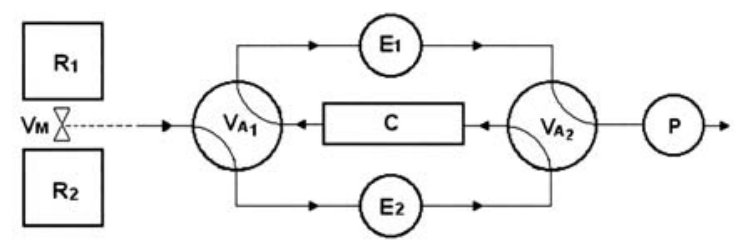

Figura 2. Diagrama general de la unidad de medida del respirómetro utilizado en este estudio: (R) Reservorios; (E) Electrodos; $\left(\mathrm{V}_{\mathrm{M}}\right)$ Válvula manual; $\left(\mathrm{V}_{\mathrm{A}}\right)$ Microválvulas automáticas (4 vías); (P) Bomba peristáltica; (C) Cámara de zooplancton. General diagram of unit meter respirometer used in this study: (R) Reservoirs; (E) Electrodes; $\left(V_{M}\right)$ Manual valve; $\left(V_{A}\right)$ Automatic microvalve (4-way); (P) Peristaltic Pump; (C) Animal chamber.

ca central; una unidad de medida (con reservorios, microválvulas de control de sentido de flujo, sensores de medida y cámaras de zooplancton) (Fig. 2); un sistema termostático de inmersión para control de la temperatura; un satélite de conexión; una bomba peristáltica y un ordenador con programas de grabación, gestión y tratamiento de datos. El sistema permite medir con elevada precisión y sensibilidad, el consumo de oxígeno entre cortos intervalos de tiempo (30 segundos) a lo largo de muchas horas (12 h.).

En las medidas de consumo de oxígeno se utilizaron ejemplares (de 3 a 8 individuos) del organismo-test, debidamente seleccionados en cuanto a la edad y mantenidos durante aproximadamente 4 horas en un recipiente (con medio M4) sin alimento, para evitar "ruidos" en las medidas. Antes de iniciar las medidas de respiración se adoptó un periodo de adaptación ( $>2$ horas) para evitar interferencias iniciales por el "stress" de los animales (debido al proceso de inserción en el dispositivo) y para los ajustes finales del sistema (flujo, renovación del medio de la cámara, etc.). Las medidas se realizaron a lo largo de periodos de 9 horas de exposición, con un flujo de medio controlado entre 1 y $2 \mathrm{ml} / \mathrm{h}$. Al final de cada serie de medidas de respiración se tomaron los valores de biomasa de los ejemplares utilizados. Los resultados fueron expresados en términos de tasa de consumo de oxígeno (tasa de respiración) por peso seco de organismo y por tiempo $\left(\mu \mathrm{l}\left(\mathrm{O}_{2}\right) \mathrm{mg}^{-1} \mathrm{~h}^{-1}\right)$.

Según Lampert (1984), el sistema de flujo abierto es especialmente utilizado para medidas 
de nivel crónico en estudios sobre efectos en las tasas de respiración por alteraciones en factores ambientales. Este sistema consiste básicamente en insertar organismos en el interior de pequeñas cámaras, por las cuales se hace pasar un flujo lento y controlado de agua o medio de cultivo, midiendo continuadamente las concentraciones de oxígeno disuelto en el flujo, a la entrada y en la salida de dichas cámaras. Las tasas de respiración son calculadas por la reducción de la concentración de oxígeno en relación al caudal del medio y la concentración de saturación del $\mathrm{O}_{2}$ (obtenida para cada momento de lectura con medidas de temperatura del medio y presión barométrica).

\section{Análisis estadístico}

Para el tratamiento inicial (almacenamiento, conversión de datos digitales, control de ficheros, etc.) se utilizó el programa informático "Datgraf 2.1 Analysis ${ }^{\odot}$ ", y para el análisis esta- dístico se empleo el programa STATISTICA (Statsoft, Inc. 1998). La evaluación de los efectos de la toxina (anatoxina-a) y la edad de los organismos sobre el consumo de oxígeno de Daphnia se llevó a cabo por medio de un ANOVA de dos vías para medidas repetidas. Se consideraron 4 periodos de medida $(0-2,2-5,5$ 8 y $8-9$ horas). Las comparaciones dos a dos se hicieron por el método LSD (Least Significant Difference). La homogeneidad de las varianzas y la normalidad se examinaron con las pruebas de Bartlett y Shapiro, respectivamente.

\section{RESULTADOS Y DISCUSIÓN}

Las tasas medias de respiración por individuo y peso seco, obtenidas en los controles con juveniles y organismos adultos, se ajustaron muy satisfactoriamente a la regresión propuesta por Lampert (1984) (Fig. 3), en la cual se

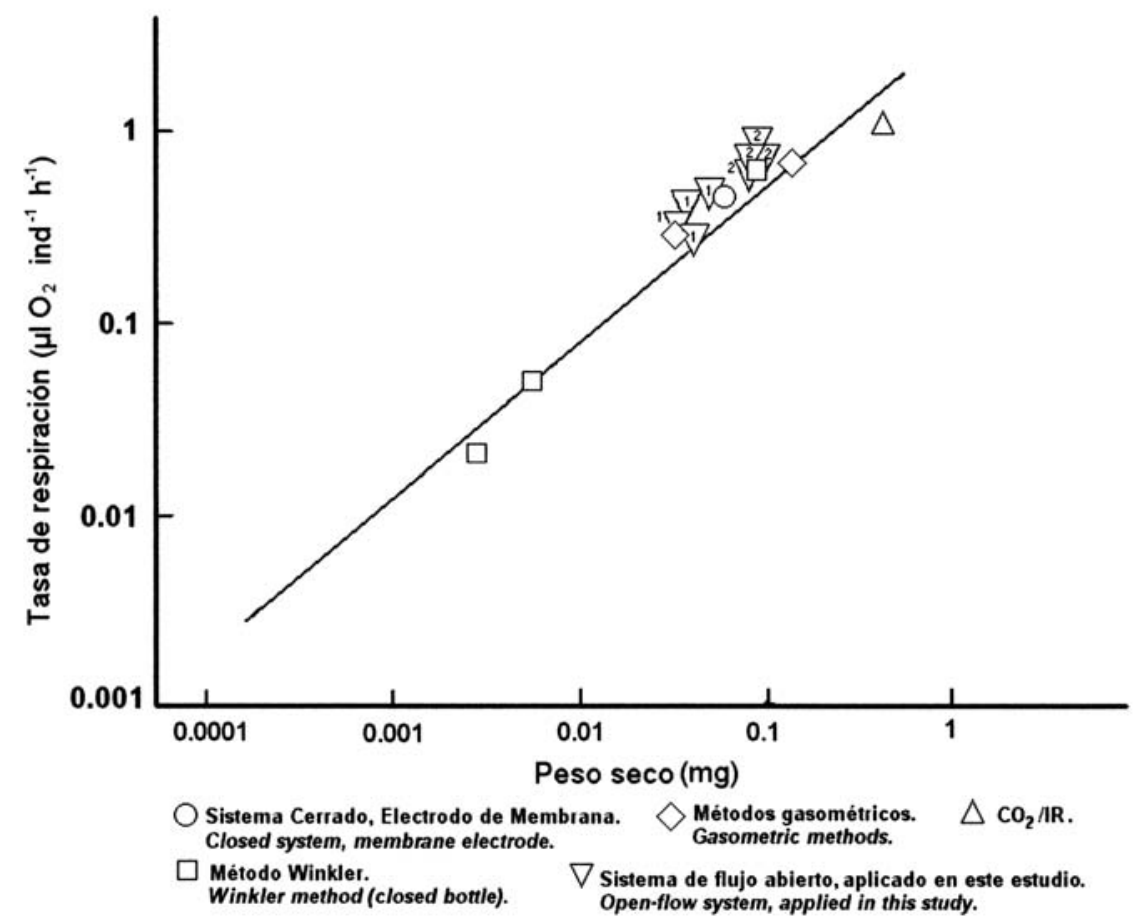

Figura 3. Tasas de respiración de Daphnia a $20^{\circ} \mathrm{C}$ (adaptado de Lampert, 1984). Los símbolos indican diferentes métodos de medidas y los números representan dos rangos de edad: 1 para organismos juveniles (0-2 días) y 2 para organismos con edad superior a 21 días. Respiratory rate of Daphnia at $20^{\circ} \mathrm{C}$ (modified from Lampert, 1984). The symbols indicate different methods of measurement and the numbers represent two range of age: 1 for juveniles (0-2 days) and 2 for animals older than 21 days. 


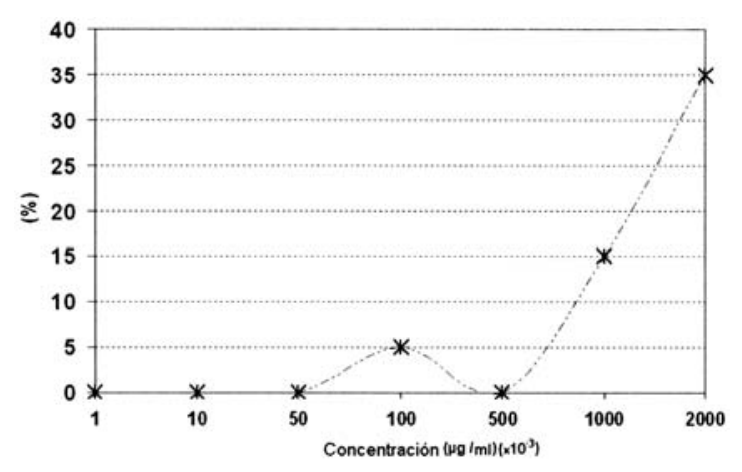

Figura 4. Inhibición de la movilidad de D. magna (\%), frente a concentraciones crecientes de anatoxina-a. Inhibition of the mobility of D. magna (\%), under increasing concentrations of Anatoxin-a.

aprecia la relación entre tasas de respiración y peso seco para distintos métodos de medida y diferentes especies del género Daphnia. Este hecho pone de manifiesto la bondad del método adoptado en este estudio.

Sobre la base del test preliminar de toxicidad realizado (Fig. 4), se estableció la concentración de $1.0 \mu \mathrm{g} / \mathrm{ml}$ para su utilización en los experimentos de consumo de oxígeno. Dicha concentración se encuentra por debajo del nivel de toxicidad aguda observado para esta especie. Por ejemplo Christoffersen (1996), recopila informaciones de tests realizados con concentraciones letales $\left(\mathrm{LC}_{50}\right)$ y subletales $\left(\mathrm{EC}_{50}\right)$ de tres cianotoxinas (microcistina, nodularina y anatoxina-a) y diferentes grupos y especies animales. Los niveles de sensibilidad observados, comprenden al menos 4 órdenes de magnitud (desde 0.001 a $20 \mu \mathrm{g} / \mathrm{ml}$ ). Crustáceos, rotíferos y peces podían ser agrupados en el rango de sensibilidad $0.5-$ $20 \mu \mathrm{g} / \mathrm{ml}$ y, concretamente, Daphnia presentó frente a anatoxina-a límites de sensibilidad comprendidos entre 3 y $10 \mu \mathrm{g} / \mathrm{ml}$.

En los experimentos de toxicidad de anatoxina-a a nivel subletal, sobre Daphnia pulex, Claska \& Gilbert (1998), utilizaron exactamente la misma concentración $(1.0 \mu \mathrm{g} / \mathrm{ml})$ que se ha empleado en nuestro trabajo.

Nuestros resultados indican que la presencia de anatoxina-a reduce significativamente el consumo de $\mathrm{O}_{2}$ de D. magna (Tabla 1).

Por lo que se conoce, esta toxina altera el funcionamiento de los neurotransmisores, actuando como una molécula de acetilcolina (ACh) en los receptores celulares del tejido muscular, pero sin sufrir degradación por la enzima acetilcolinesterasa (AChE), lo que provoca una continuada contracción del tejido muscular, con la consecuente pérdida de sus funciones. Los animales afectados mueren por asfixia al paralizar los músculos respiratorios. Aunque no se hayan encontrado informaciones específicas en cuanto al posible mecanismo de acción en cladóceros, algunos trabajos (p.ej. Hyne \& Maher, 2001) han demostrado que determinadas especies de crustáceos dulceacuícolas sufren una considerable inhibición de la actividad de la acetilcolinesterasa en exposición a insecticidas organofosforados, por mecanismos semejantes al provocado por otra forma de neurotoxina: la anatoxina-a(s).

Tabla 1. Resultados del Análisis de Varianza de dos vías con medidas repetidas; * Diferencias significativas $(\mathrm{P}<0.05)$. Results of two-way Analyses of Variance with repeated measures; * Significant differences $(P<0.05)$.

\begin{tabular}{lrrrr}
\hline & d.f & MS & F & p \\
\hline anatoxina-a & 1 & 141.009 & 34.75606 & $<0.001 *$ \\
Edad & 1 & 0.285 & 0.00703 & 0.935 \\
anatoxina-a $\times$ edad & 1 & 109.840 & 27.07348 & $0.001 *$ \\
Error & 12 & 4.057 & 1.00607 & 0.401 \\
Tiempo & 3 & 1.351 & 0.04399 & 0.988 \\
anatoxina-a $\times$ tiempo & 3 & 0.591 & 2.28533 & 0.953 \\
Edad $\times$ Tiempo & 3 & 3.069 & 0.05417 & 0.983 \\
anatoxina-a $\times$ edad $\times$ tiempo & 3 & 0.073 & & \\
Error & 36 & 1.343 & & \\
\hline
\end{tabular}



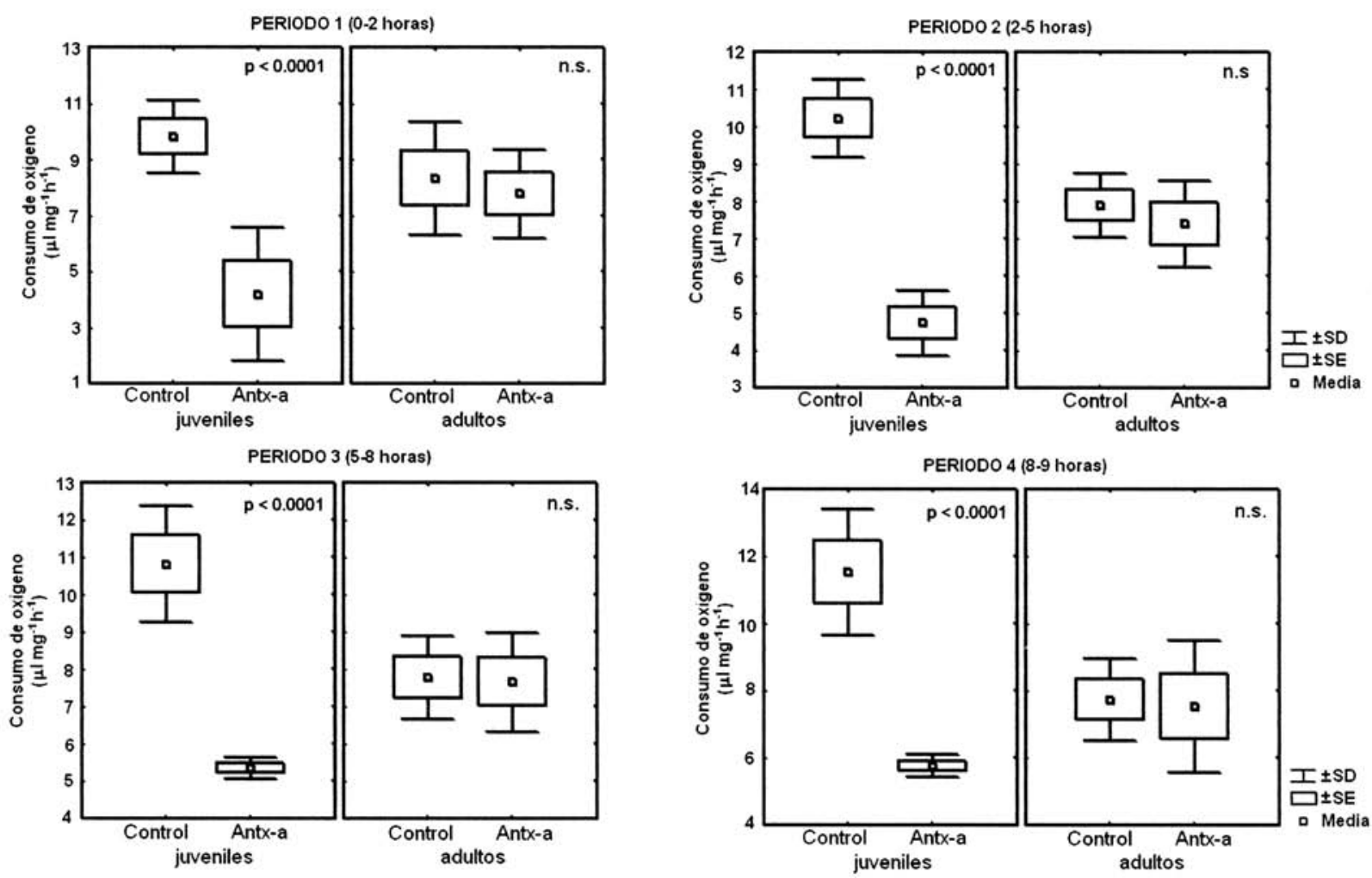

Figura 5. Media, desviación estándar (SD) y error estándar (SE) del consumo de oxígeno, en los diferentes tratamientos - con anatoxina-a (Antx-a), sin anatoxina-a (Control) - edades juveniles y adultos. Las comparaciones entre tratamientos se realizaron con análisis LSD. n.s.: no significativo. Mean, standard deviation (SD) and standard error (SE) of the oxygen consumption for the different treatments - with Anatoxin-a (Antx-a), without Anatoxin-a (Control) - juveniles and adults. Comparisons between treatments were performed by using the LSD test. n.s.: non significant.

Además nuestros resultados ponen de manifiesto que la interacción toxina-edad tuvo un efecto significativo (Tabla 1). De hecho, observamos que el consumo de oxígeno se reduce significativamente más en los individuos juveniles que en los adultos en presencia de la anatoxina-a (Fig. 5), sugiriendo que los individuos juveniles de $D$. magna son más susceptibles a esta toxina. Guilhermino et al. (1996) observaron que individuos juveniles de D. magna (bajo condiciones de laboratorio) presentaron mayores niveles de la enzima AChE que los organismos adultos. Nuestros resultados obtenidos en los tratamientos controles ( $\sin$ anatoxina-a), ponen de manifiesto que las diferencias en las tasas de respiración entre juveniles y adultos, han sido casi siempre significativamente $(\mathrm{p}<0.05)$ diferentes
(Fig. 6), presentando los juveniles una mayor tasa de consumo de oxígeno. Estos elevados niveles de AChE y consumo de oxígeno en los juveniles, en relación con los organismos adultos, pueden significar la correspondiente existencia de una elevada actividad de ACh y como quiera que la anatoxina-a se une y actúa en los terminales nerviosos, en lugar de la $\mathrm{ACh}$, esto podría significar en ultimas instancias, una mayor susceptibilidad al efecto de dicha cianotoxinas en estos individuos juveniles.

Comparaciones de estos resultados con concentraciones de anatoxina-a, tomadas a partir de "blooms" de cianobacterias, serían poco factibles, toda vez que dichas medidas son poco frecuentes y presentan muy variados valores, con concentraciones que van desde 0.3 hasta 4 

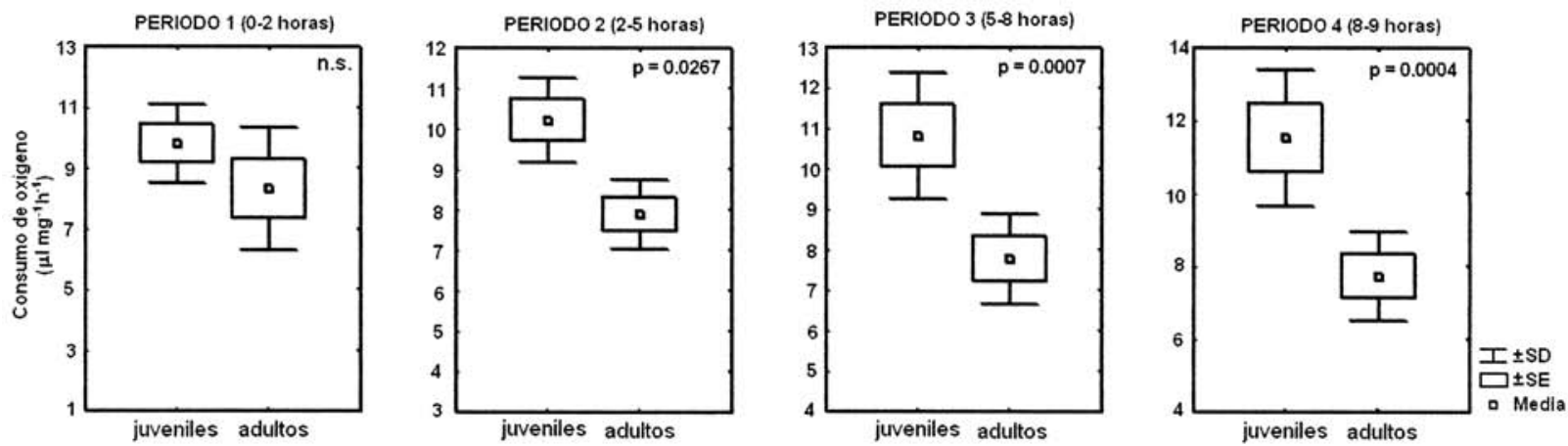

Figura 6. Media, desviación estándar (SD) y error estándar (SE) del consumo de oxígeno, en los controles, entre dos edades (juveniles y adultos). Las comparaciones entre tratamientos se realizaron con análisis LSD. n.s.: no significativo. Mean, standard deviation (SD) and standard error (SE) of the oxygen consumption in the control treatments, between two ages (juveniles and adults). Comparisons between treatments were performed by using the LSD test. n.s.: non significant.

$400 \mu \mathrm{g} \mathrm{g}^{-1} \mathrm{dw}$ (de cianobacteria) (Sivonen \& Jones, 1999). La variabilidad observada en dichas medidas, puede ser resultado, por una parte, de diferencias metodológicas en cuanto a la toma de muestras y detección y, por otra, de la variabilidad en los factores físicos y bióticos que estimulan y en cierta medida pueden controlar la producción de las cianotoxinas (Christoffersen, 1996). Bajo condiciones "in vitro", medidas de anatoxina-a en cultivos de Anabaena flos-aquae (Bumke-Vogt et al., 1996), alcanzaron concentraciones de $1600 \mu \mathrm{g} / 1$ (medio) con 5 semanas y con una masa celular equivalente a $500 \mathrm{mg}(\mathrm{dw}) / 1$.

De cualquier manera, la anatoxina-a es una potente neurotoxina producida por especies de Anabaena, Oscillatoria y Aphanizomenon (Sivonen, 1998). Por lo tanto, en aquellos sistemas naturales donde estas especies se desarrollen y produzcan dicha cianotoxina, los efectos de ésta sobre el zooplancton serán de importancia variable dependiendo, entre otros, del estado de desarrollo de las poblaciones individuales.

\section{AGRADECIMIENTOS}

Este trabajo ha sido financiado por el proyecto CICYT HID 99-0836.

\section{BIBLIOGRAFIA}

ABNT NBR 12713. 1993. Ensaio de toxicidade aguda com Daphnia similis Claus, 1876 (Cladocera, Crustacea). Associação Brasileira de Normas Técnicas, 16 pp.

ABNT NBR 12648. 1992. Ensaio de toxicidade com Chlorella vulgaris (Chlorophyceae). Associação Brasileira de Normas Técnicas, 8 pp.

AENOR UNE-EN ISO 6341. 1996. Determinación de la inhibición de la movilidad de Daphnia magna Straus (Cladocera, Crustacea). Asociación Española de Normalización y Certificación. 17 pp.

BARROS, P. 1994. Implicações ecotoxicológicas de cianobactérias em cladóceros. Tésis de Mestrado, Universidade de Coimbra. 84 pp.

BARROS, P., M. L. FIDALGO, A. M. V. M. SOARES. 2001. Resistance of Cladoceran Species to Toxic Microcystis. Limnetica, 20: 173-177.

BUMKE-VOGT, C., W. MAILAHN, W. ROTARD \& I. CHORUS. 1996. A highly sensitive analytical method for the neurotoxin anatoxin-a, using GCECD, and first application to laboratory cultures. Phycologia, 35(6): 42-50.

CARMICHAEL, W. W. 1994. The Toxins of Cyanobacteria. Sci. Am., 270: 78-86.

CHRISTOFFERSEN, K. 1996. Ecological implications fo cyanobaterial toxins in aquatic food webs. Phycologia, 35(6): 42-50.

CLASKA M. E. \& J. J. GILBERT. 1998. The effect of temperature on the response of Daphnia to toxic cyanobacteria. Freshwater Biol., 39: 221-232. 
CRUZ-PIZARRO, L., M. V. AMORES, D. FABIAN, I. DE VICENTE, I. RODRÍGUEZ-PARÍS, K. EL MABROUKI, M. RODRÍGUEZ-RODRÍGUEZ y S. L. RODRIGUES DA SILVA. 2002. La Eutrofización de las Albuferas de Adra. 1as Jornadas de Formación Agrícola: Agricultura y Medio Ambiente en el Entorno de Albuferas de Adra, Almería, España: 77-96.

DAVIDOWICZ, P., Z. M. GLIWICZ \& R. D. GULATI R. 1988. Can Daphnia Prevent a Blue-green Algal Bloom in Hypertrophic Lakes? A Laboratory Test. Limnologica (Berlin), 19: 21-26.

ELENDT, B. \& W. R. BIAS. 1990. Trace nutrient deficiency in Daphnia magna cultured in standard medium for toxicity testing. Effects of the optimization of culture conditions on life history parameters of Daphnia magna. Wat. Res., 24(9): 1159-1167.

FULTON, R. S. \& H. W. PAERL. 1987. Toxic and inhibitory effects of the blue-green alga Microcystis aeruginosa on herbivorous zooplankton. J. Plankton Res., 9: 837-855.

GLIWICZ, Z. M. \& W. LAMPERT. 1990. Food Thresholds in Daphnia Species in the Absence and Presence of Blue-green Filaments. Ecology, 71(2): 691-702.

GUILHERMINO, L., M. C. LOPES, A. P. CARVALHO, A. M. V. M. SOARES. 1996. Acetylcholinesterase Activity in Juveniles of Daphnia magna Straus. Bull. Environ. Contam. Toxicol., 57: 979-985.
HYNE, R V. \& W. A. MAHER. 2001. Macroinvertebrate Biomarkers: Links to Toxicosis and Changes in Populations or Communities. Coop. Research Centre Freshwater Ecology. Australia. 23 pp.

INTERNATIONAL ORGANIZATION FOR STANDARDIZATION. 1982. Water quality Determination of the inhibition of the mobility of Daphnia magna Straus (Cladocera, Crustacea). ISO 634. First edition. 9 pp.

LAMPERT, W. 1984. The Measurement of Respiration. In: A Manual of Methods for the Assessment of Secondary Productivity in Fresh Waters. $2^{\text {nd }}$ ed. Downing, J. A. \& Rigler, F. H. (eds).:413-468. Blackwell Scientific Publications. Boston.

LAMPERT, W. 1987. Laboratoy studies on zooplankton-cyanobacteria interactions. New Zeland J. Mar. Fresh. Res., 21: 483-490.

SIVONEN, K. 1998. Toxin Produced by Cyanobacteria. In: Mycotoxins and Phycotoxins Developments in Chemistry, Toxicology and Food Safety. M. Miraglia, H. van Egmond, C. Brera \& J. Gilbert (eds.): 547-568. Alaken, Inc., Fort Collins, CO, USA.

SIVONEN, K. \& G. JONES, G. 1999. Cyanobacterial toxins. In: Toxic Cyanobacteria in Water Chorus, I. \& Bartram, J. (eds.): 41-111. E \& F.N. Spon. London.

VASCONCELOS, V. M. 2001. Cyanobacteria Toxins: Diversity and Ecological Effects. Limnetica, 20: 45-58. 